the VRE case-patients and the vancomycin-susceptible enterococcal control-patients, but case-patients had higher Acute Physiology and Chronic Health Evaluation II scores and received significantly greater numbers of antimicrobials and significantly more days of antimicrobials during the 60 days preceding the first positive enterococcal culture. In an assessment of the appropriateness of vancomycin use, one third of vancomycin orders were found to be inappropriate in both patient groups.

The authors concluded that that among renal patients, those who are severely ill and receive multiple and prolonged courses of antimicrobials are at greatest risk for acquiring VRE infection or colonization.

FROM: Beltrami EM, Singer DA, Fish L, Manning K, Young S, Banerjee SN, et al. Risk factors for acquisition of vancomycin-resistant enterococci among patients on a renal ward during a community hospital outbreak. $A m J$ Infect Control 2000;28:282-285.

\section{Bacterial Colonization of Toys in Neonatal Intensive Care Cots}

Davies and coinvestigators from the Royal Women's Hospital, Melbourne, Australia, conducted a study to investigate which bacteria and fungi contaminated toys in neonatal ICU (NICU) cots. A cross-sectional, longitudinal, bacteriologic survey was conducted of all toys in the cots of infants in an NICU. Cultures of toys were obtained weekly for 4 weeks. Data were collected on the infant's postnatal age, the type of cot, whether humidity was added, characteristics of the toy, and any infant infections.

Over the 4-week period, there were 86 cultures from 34 toys of 19 infants. Bacteria were grown from $84(98 \%)$ of 86 cultures: 84 grew coagulase-negative staphylococci; 50 , Micrococcus species; 21, Bacillus species; 13, methicillinresistant Staphylococcus aureus; 12 , diphtheroids; 4 , group B streptococcus; $3, S$ aureus; 3 , nonhemolytic streptococci; 3 , group D streptococci; 4, alpha-hemolytic streptococci; and 2, coliforms. None grew fungi. The colonization rate did not differ with cot type, presence of humidity, size of the toy, toy fiber length, or the fluffiness score. Eight (42\%) of the infants had positive blood culture results, and $5(63 \%)$ of the 8 isolates were of the same type as that colonizing their corresponding toy.

The authors point out that, with time, all the toys in NICU cots became colonized with bacteria. Many were potentially pathogenic. Toys may be reservoirs for potential infantile nosocomial sepsis.

FROM: Davies MW, Mehr S, Garland ST, Morley CJ. Bacterial colonization of toys in neonatal intensive care cots. Pediatrics 2000;106:E18.

\section{Prolonged Viremia With Hepatitis A Virus Infection}

Researchers from the CDC recently reported on the results of a study to determine the duration of viremia in hepatitis A virus (HAV) infection and the onset of IgM antibodies to HAV. The duration of viremia and time course for development of IgM antibodies were determined prospectively in natural and experimental HAV infection. Serial serum samples from $13 \mathrm{HAV}$-infected men and 5 experimentally infected chimpanzees were examined by nested reverse-transcriptase polymerase chain reaction analysis to detect HAV RNA and by enzyme-linked immunosorbent assay to detect IgM antibodies to HAV. Among infected humans, HAV RNA was detected an average of 17 days before the alanine aminotransferase peak, and viremia persisted for an average of 79 days after the liver enzyme peak. The average duration of viremia was 95 (range, 36-391) days. Results were similar in chimpanzees. In addition, HAV RNA was detected in serum of humans and chimpanzees several days before IgM antibodies to HAV were detected. These results indicate that adults with HAV infection are viremic for as long as 30 days before the onset of symptoms and that the duration of viremia may be longer than previously described.

The authors note that these findings have important implications for the transmission of HAV infection through blood products. Blood collected before the onset of symptoms appears to be at highest potential risk of transmitting HAV infection because of the high concentration of virus and essentially no antibody.

FROM: Bower WA, Nainan OV, Han X, Margolis HS. Duration of viremia in hepatitis A virus infection. $J$ Infect Dis 2000;182:12-17.

\section{Hepatitis B Outbreak Linked to Autohemotherapy}

Webster and colleagues recently reported on a lookback investigation conducted among patients who received treatment at an autohemotherapy clinic in London following diagnosis of acute hepatitis B virus (HBV) infection in a patient who had recently been treated at the clinic. The HBV outbreak investigation was led by the local public health authority and the Public Health Laboratory Service Communicable Disease Surveillance Centre, who identified 399 patients attending the clinic between January 1997 and September 1998. Autohemotherapy is available in alternative medicine clinics worldwide; patients seek treatment for a variety of ailments, including allergies, malignancy, viral hepatitis, and herpes zoster. The autohemotherapy procedure involved drawing approximately $1 \mathrm{~mL}$ of the patient's blood with a needle and syringe, mixing the blood with an equal volume of saline, and injecting the mixture into the buttocks or acupuncture points.

Transmission of HBV was linked to a multiuse vial of saline that was drawn from after each venipuncture with the syringe containing the patient's blood. The authors state that the point source of infection in this outbreak could not be unequivocally identified, but one of five patients who were classified as having chronic HBV infection was regarded as the likely source of the infec- 